\title{
Les réponses oraculaires dans la Division des questions de Sopatros
}

Julie Dainville

\section{OpenEdition}

12 Journals

Édition électronique

URL : https://journals.openedition.org/rhetorique/1244

DOI : 10.4000/rhetorique.1244

ISSN : 2270-6909

Éditeur

UGA Éditions/Université Grenoble Alpes

Édition imprimée

ISBN : 978-2-37747-325-0

\section{Référence électronique}

Julie Dainville, "Les réponses oraculaires dans la Division des questions de Sopatros », Exercices de rhétorique [En ligne], 17 | 2021, mis en ligne le 25 novembre 2021, consulté le 29 novembre 2021. URL : http://journals.openedition.org/rhetorique/1244 ; DOI : https://doi.org/10.4000/rhetorique.1244

Ce document a été généré automatiquement le 29 novembre 2021.

\section{(c) (1) () (2)}

Les contenus de la revue Exercices de rhétorique sont mis à disposition selon les termes de la Licence Creative Commons Attribution - Pas d'Utilisation Commerciale - Partage dans les Mêmes Conditions 4.0 International. 


\title{
Les réponses oraculaires dans la Division des questions de Sopatros
}

\author{
Julie Dainville
}

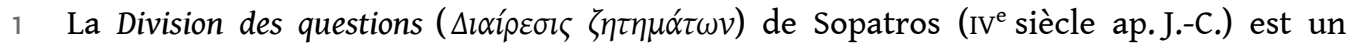
ouvrage fondamental pour notre connaissance de la déclamation grecque. Orienté vers la pratique, il contient 82 énoncés, classés par état de cause, ainsi que les recommandations du rhéteur, accompagnées d'exemples de développement, pour diviser les cas en question. Le lecteur trouvera, dans ce qui suit le texte et la traduction, des questions mentionnant une réponse oraculaire ${ }^{1}$. L'objectif de cette publication est de mettre en avant ces thèmes, particulièrement riches pour notre compréhension de la relation complexe qu'entretenait rhétorique et divination dans l'Antiquité2.

2 Sauf mention contraire, je reproduis dans ce qui suit l'édition établie par M. WEISSENBERGER ( Sopatri Quaestionum Divisio. Sopatros, Streitfälle. Gliederung und Ausarbeitung kontroverser Reden, Herausgegeben, übersetzt, mit einer Einleitung une einem Glossar versehen, Würzburg, Königshausen et Neumann, 2010), ainsi que sa numérotation des énoncés.

\section{Sopatros, Division des questions [extraits avec traduction et notes]}

\section{Discours d'excuse ( $\sigma u \gamma \gamma v \omega ́ \mu \eta^{3} ; 8,232,22-238,21$ Walz)}

3 Une cité frappée par la maladie a envoyé son général consulter les dieux, afin de savoir comment en venir à bout. Le dieu a répondu que si le fils du général était sacrifié, la cité serait libérée de la maladie. Une fois rentré, il a seulement annoncé que le dieu ordonnait un sacrifice. Son fils, après avoir eu connaissance de l'oracle, s'est suicidé. L'épidémie a pris fin et le général est jugé pour faute envers l'État.

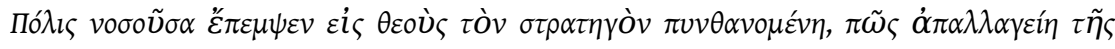

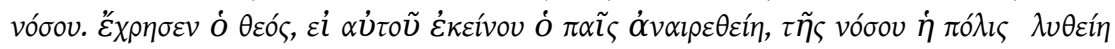




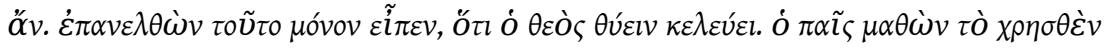

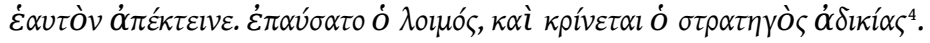

\section{Défense ${ }^{5}$}

4 L'objectif pour l'accusateur n'est pas seulement de faire condamner le général, mais aussi qu'il soit destitué de sa charge. C'est pourquoi, par crainte de cela, le général établira autant que possible qu'il ne faut pas le destituer de sa charge. Il procédera en ce sens en énumérant exhaustivement ses prouesses, en faisant valoir qu'il n'a jamais été condamné et que la cité n'a jamais été vaincue, mais qu'au contraire, il a protégé les enfants, les femmes, les hommes dans la force de l'âge, les vieillards, et que, sous son commandement, aucun ennemi n'a jamais pris de trophée, de trières, de prisonniers, ou quoi que ce soit de tel. Grâce à ces éléments et à d'autres similaires, il établira clairement qu'il ne faut pas lui retirer son commandement. Le discours sera pathétique et plein de convenance en raison du chef d'accusation et de l'acte. L'accusation utilisera autant que possible les arguments qui vont dans le sens contraire.

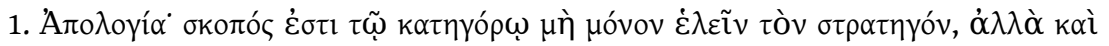

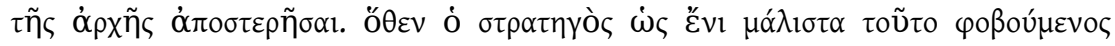

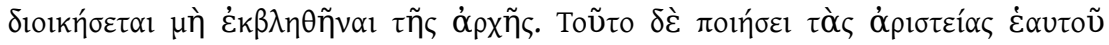

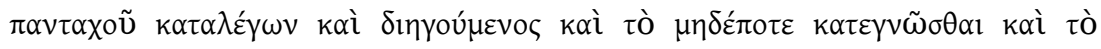

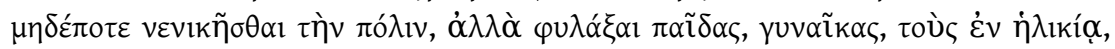

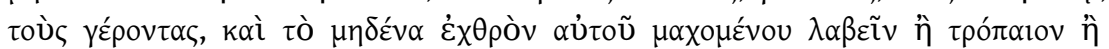

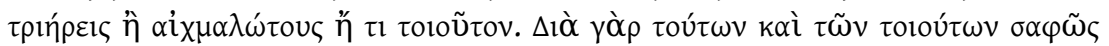

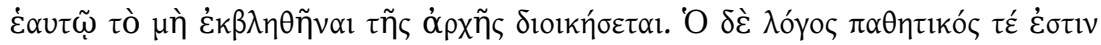

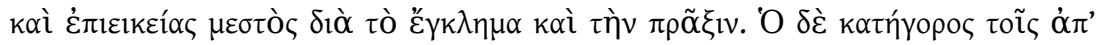

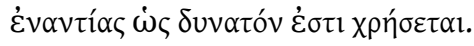

\section{L'exposé en faveur du général}

Il comprend son élection confirmée par la docimasie ${ }^{6}$ et l'admiration pour son dévouement qui a mené à son élection en tant que général. Ensuite les combats, les victoires, les trophées, avec amplification. Ensuite, avec gravité et évocation, la maladie, mais sans proposer, à aucun moment, le récit des malheurs causés par la maladie et sans décrire les souffrances qu'elle a causées ${ }^{7}$. Cela joue en effet en sa défaveur si, alors que les souffrances étaient si nombreuses, il n'a pas rapporté l'oracle dans son intégralité. Mais l'accusation le fera, tandis que le général ne les utilisera que sommairement. Ensuite, la question au dieu et l'oracle, avec une éthopée pleine de douleur et d'émotion : " je fus frappé de stupeur, lorsque j'ai entendu l'oracle, et je fus plongé dans le malheur ", etc. Ensuite: "Accablé, gémissant, en larmes, pleurant mon enfant je suis rentré chez moi, j'ai annoncé notre sort, pensant qu'un simple sacrifice apaiserait le dieu ». Il faudra en effet que tu établisses en détail pourquoi il n'a annoncé qu'un simple sacrifice. Ensuite : «Mon fils, je ne sais comment, a appris l'oracle et a délibéré d'une manière digne de l'ambition de son père. Si moi, en effet, la nature m'a empêché de délibérer de la sorte sur cette question, lui n'a pas déshonoré sa lignée : il s'est sacrifié de lui-même pour les enfants, les femmes, pour la patrie tout entière. Et il gît là comme s'il était tombé au combat, alors que son seul combat fut contre ce fléau », etc. "C'est contre cela qu'est intenté le procès, c'est contre cela qu'est portée l'accusation, ou plutôt : le procès est intenté contre la nature; l'accusation, contre la condition humaine ». Et : "J'ai été contraint d'aimer ma famille à cause de la nature ", etc. ${ }^{8}$ 


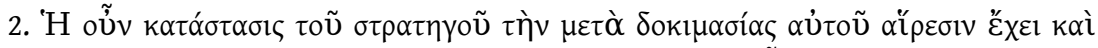

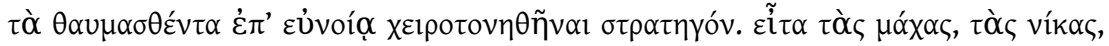

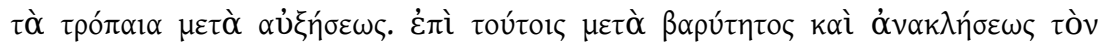

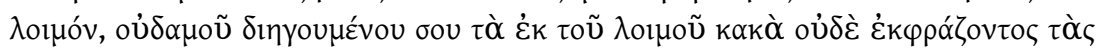

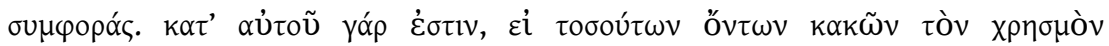

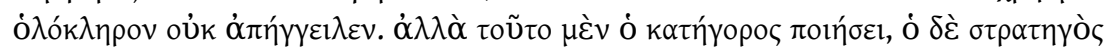

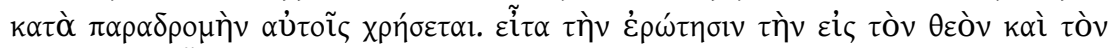

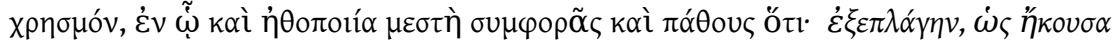

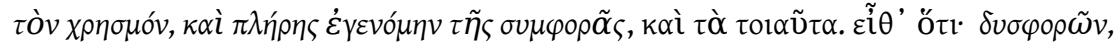

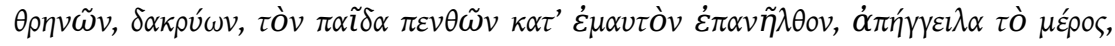

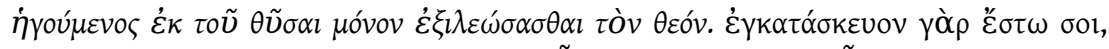

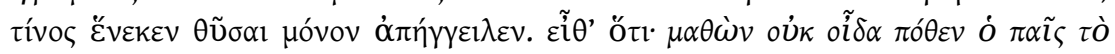

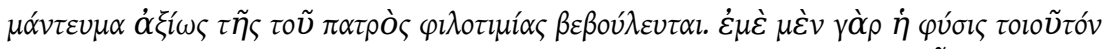

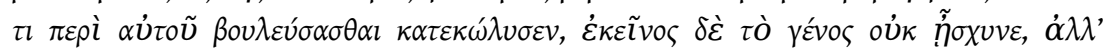

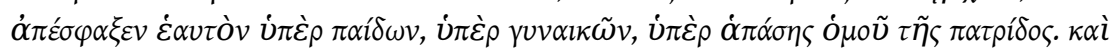

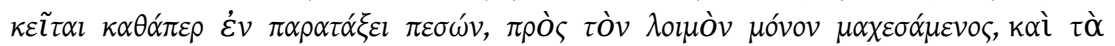

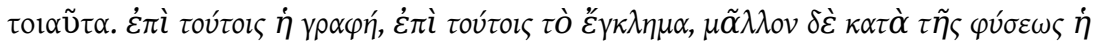

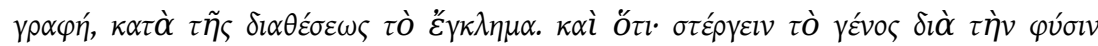

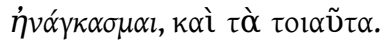

\section{3. [Compte-rendu des événements]}

6 Après cela, il n'y a pas de procédure d'exception fondée sur la définition ${ }^{9}$, mais tu en viendras directement, après la narration, au compte-rendu des événements ${ }^{10}$ : «tu ne nous as pas rapporté, dit-il, l'oracle complet ». La réponse en vue de l'obtention du pardon: "j'ai eu pitié de mon fils et, confronté à ce malheur, je n'ai pas consenti à dire le reste de la réponse ». Et : " face aux malheurs, les hommes sont tous naturellement disposés à ne plus être eux-mêmes et à se consacrer corps et âme à leurs souffrances ». Et : "l'enfant était mon seul soutien dans la vieillesse et j'espérais qu'il serait mon successeur, mon malheur fut donc plus grand encore ", etc. Tu diras certaines choses d'un ton sentencieux, et d'autres en dépeignant les espoirs du père : il était son seul enfant; tu tireras argument de la nature : nous sommes tous naturellement disposés à très difficilement supporter les malheurs qui frappent les nôtres. Et : je suis général, je combats pour le salut de tous les enfants et je leur assure par mes victoires d'être toujours saufs : cette fois, c'est avant tout mon propre enfant que je voulais voir sauf. Ensuite: Et maintenant, cette pensée m'est insupportable. Que le discours en effet soit plein d'émotion.

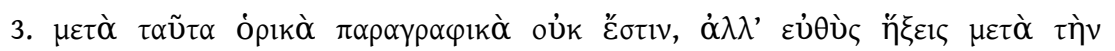

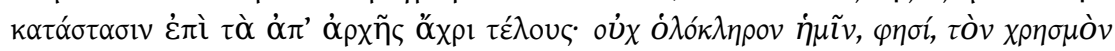

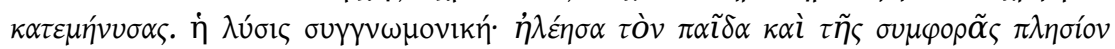

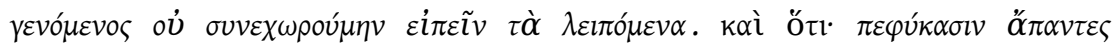

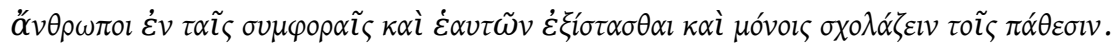

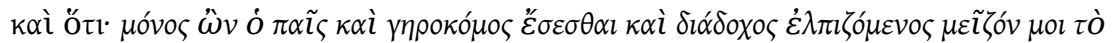

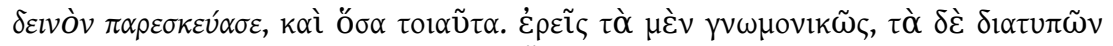

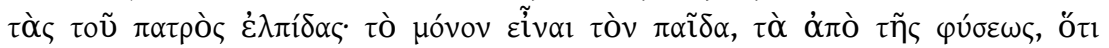

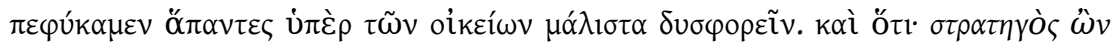

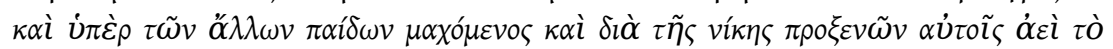

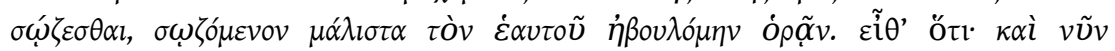

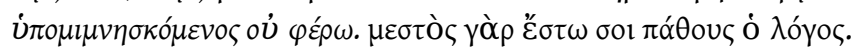




\section{4. [Arguments : l'intention]}

7 Ensuite, après cela, avance les arguments tirés de l'intention du général ${ }^{11}:$ «il est clair, en effet, qu'il a gardé le silence parce qu'il a pris en pitié son enfant et s'est lamenté sur son sort, et parce qu'il souhaitait son salut. Et si cela n'est pas vrai, pour quelle raison se serait-il tu? » Dans cette partie, tu énuméreras avec zèle toutes les qualités du général : il est bienveillant, attaché à son peuple et à sa cité, il occupe la charge de général, il a remporté des victoires, et tout ce qu'il est possible de tirer des lieux de l'éloge pour établir son intention: je me suis tu parce que je pensais à mon fils, et aucunement par méchanceté. Ensuite, argumente aussi à partir de l'intention prêtée par l'adversaire. Si en effet ce n'est pas vrai, me suis-je un jour montré méchant? ou malveillant? Ai-je commis quelque acte répréhensible? Ensuite avance encore un argument agonistique: «si je n'ai jamais été méchant, il est clair que je ne le suis pas non plus maintenant ", etc.

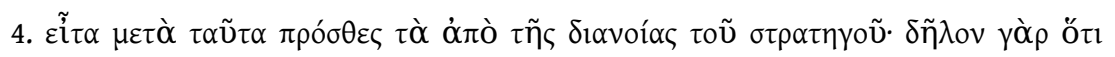

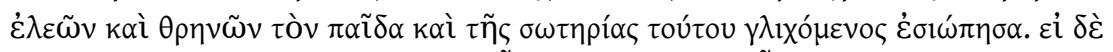

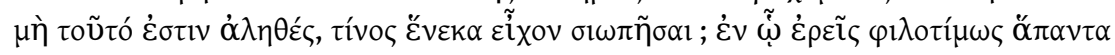

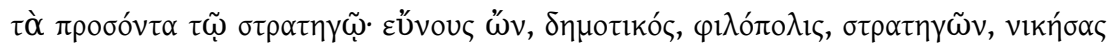

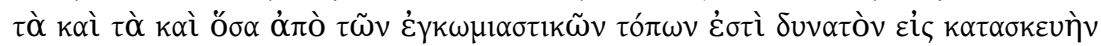

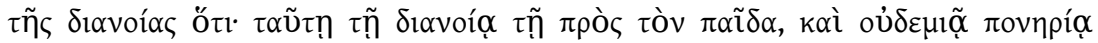

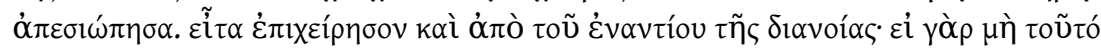

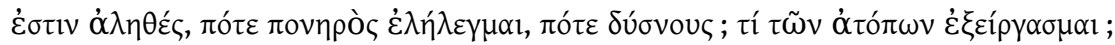

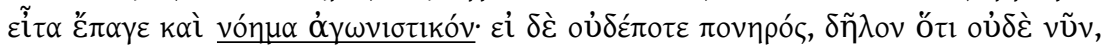

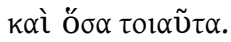

\section{5. [Arguments : la définition forcée]}

Ensuite, après l'intention, avance la définition forcée ${ }^{12}:$ " et c'est en cela même que consiste le fait de ne pas nuire à la cité ». Et avance un argument plus forcé encore : « l'enfant étant clairement une partie de la cité, si j'ai causé du tort, je n'ai pas causé du tort à la cité tout entière. Comment donc ? Pour la défense de mon enfant, qui est aussi une partie de la cité, j'ai fait preuve de prévoyance ", etc.

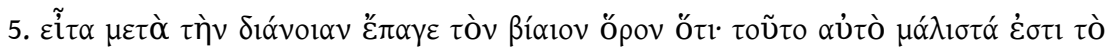

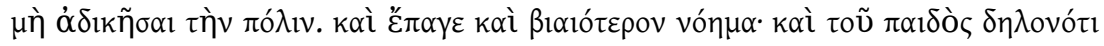

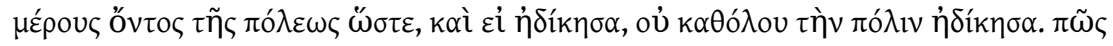

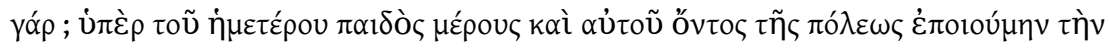

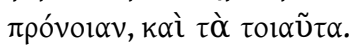

\section{6. [Compte-rendu des événements : suite]}

Après cela intervient la suite du compte-rendu des événements, selon la circonstance et la matière ${ }^{13}$. En effet, puisque le fils s'est lui-même sacrifié après avoir appris l'oracle, il répétera cela en réponse à la demande de pardon: "mais c'était là ce que souhaitait l'enfant ». Tu réfuteras à nouveau en cherchant à obtenir le pardon : «j'ignorais vraiment si la mort était ce que souhaitait mon fils ou pas. Comment aurais-je pu le savoir, puisque tous les hommes fuient la mort? Ensuite, même si je l'avais su, je craignais surtout ici d'être privé de mon enfant, mon seul enfant, qui administrait seul cette maison, m'assistait, me réconfortait dans les difficultés, m'épaulait dans les situations délicates, se réjouissait avec moi dans les succès ». Ensuite : " et nous sommes tous enclins à éprouver de la peine à nous séparer de notre famille, en particulier de nos enfants. Aussi, étant dans une telle disposition, comment aurais-je pu annoncer l'oracle? Comment aurais-je pu me montrer ferme alors que je me représentais 
l'enfance de mon fils, son éducation, sa conduite, la séparation, sa mort? Ces pensées m'ont disposé au silence, ces pensées ont fait que je n'ai pas révélé l'oracle dans son intégralité ", etc.

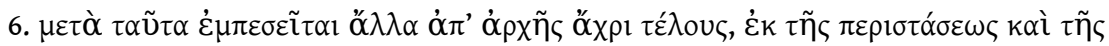

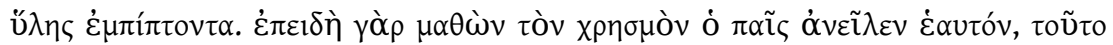

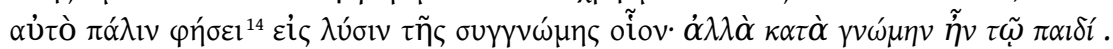

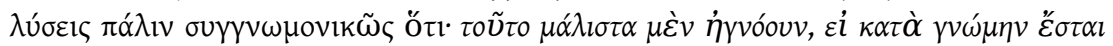

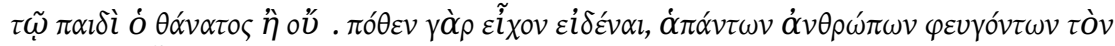

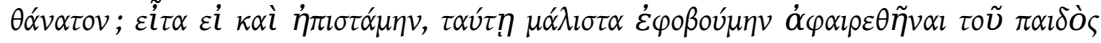

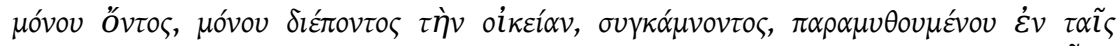

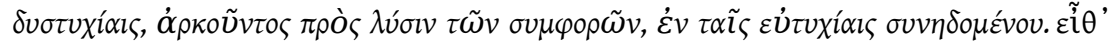

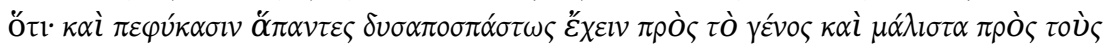

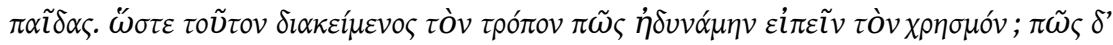

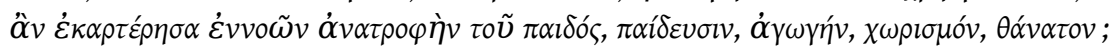

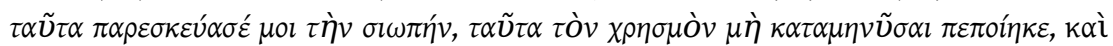

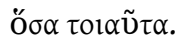

\section{7. [Arguments, suite]}

10 Après cela tu poseras la métalepse : « mais il fallait, dit-il, faire passer la cité avant ». La réponse tirée des circonstances sur le mode de la concession ${ }^{15}:$ «je le reconnais moimême : en effet, j'ai également été élevé ainsi et j'ai transmis ces valeurs à mon fils. Mais la nature est trop contraignante ». Et: "c'est elle qui dirige et qui gouverne absolument tout». Et: «c'est elle qui m'a contraint». Ensuite à partir d'exemples démontre ceci: "nous voyons que les bêtes sauvages et tous les animaux sont gouvernés par la nature, ils font passer leur famille avant tous les autres à cause de leur nature, ils se battent désespérément en raison de la nature et se mettent souvent en danger », etc. Ensuite tu diras : «j'ai moi-même, de la même manière, été gouverné par la nature, elle m'a contraint à emprunter cette voie ». Et : « nous sommes nombreux à avoir commis de nombreuses erreurs de jugement en raison de la nature: l'un a souvent dédaigné les punitions pour faire plaisir à son enfant, l'autre est capable de renoncer à tout pour le salut de son enfant ». Et : «être disposé ainsi est conforme à notre nature, car nos enfants sont une partie de nous », etc. Tu continueras en passant en revue les arguments tirés, d'une part, de la nature, d'autre part, des lois, à partir d'exemples, et à partir de parallèles. Et tu trouveras de nombreux arguments pour cela.

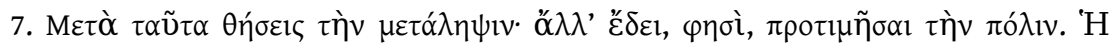

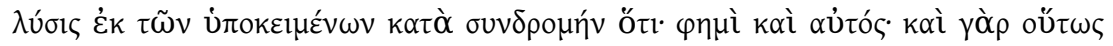

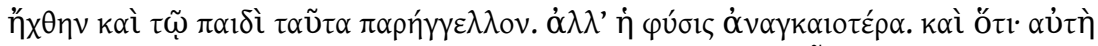

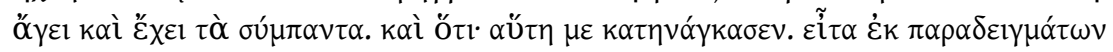

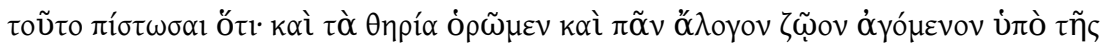

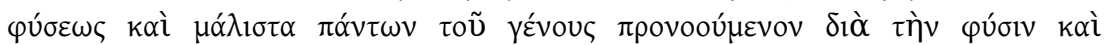

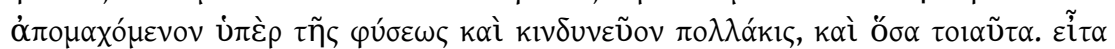

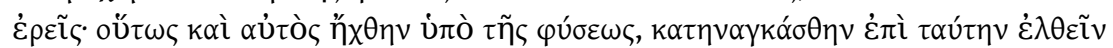

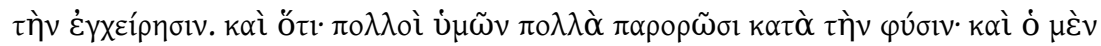

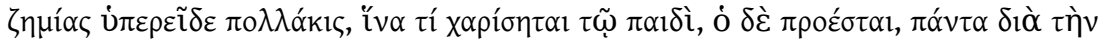

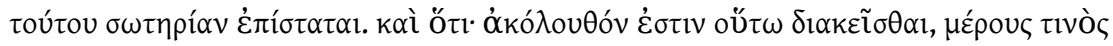

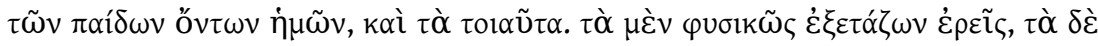

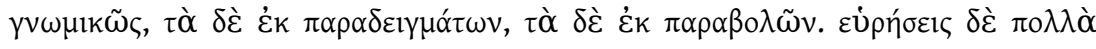

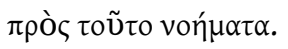




\section{8. [Arguments, suite]}

11 Ensuite tu poseras une autre métalepse : « mais il fallait que le messager demande à la cité de consulter le dieu une nouvelle fois ». La réponse sur le mode de la réfutation: "personne ne m'aurait accordé de procéder ainsi et vous n'auriez pas accepté, même si je vous avais priés et suppliés de consulter le dieu à nouveau ». Et : «ce fléau et la maladie ne m'auraient pas donné une seule occasion de vous implorer, alors qu'un homme gisait là, mort, et qu'un autre était sur le point de succomber ». Ensuite : « Tous ceux qui étaient malades se seraient dressés autour de moi en signe de contestation et auraient refusé d'être affligés plus longtemps par la maladie, en attendant que je sois à nouveau envoyé pour consulter le dieu». Ensuite : «et personne parmi vous n'aurait jugé indécent qu'un seul enfant meure pour le salut de tous, pour le salut des enfants, des femmes, des vieillards ", etc.

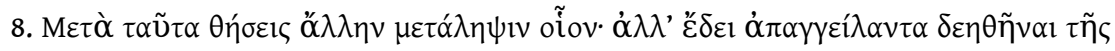

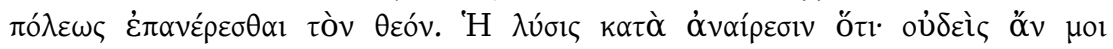

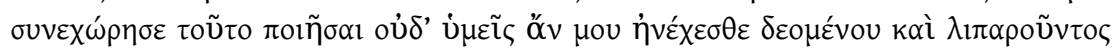

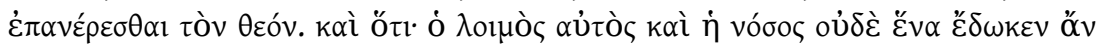

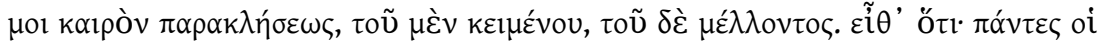

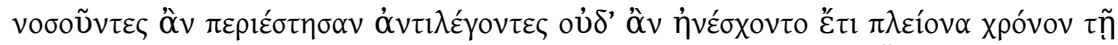

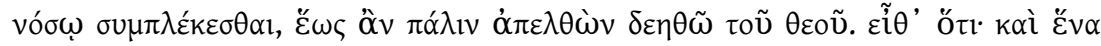

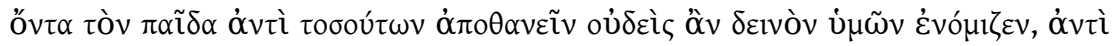

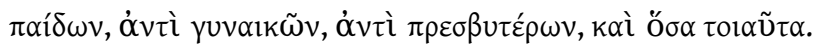

\section{9. [Épilogues]}

Ensuite, les épilogues du général : «j'ai fait beaucoup pour vous, pour vos enfants, pour vos femmes »; et : «surtout, j'ai assuré le salut de beaucoup d'entre vous ». Et: « je ne me suis jamais laissé aveugler par mon intérêt personnel, je n'ai jamais perdu un combat, et grâce à moi, aucun de vos enfants n'a connu la servitude ». Grâce à de tels propos, tu feras en sorte qu'il ne soit pas démis de ses fonctions. Ensuite tu diras: "mais puisque mon unique enfant est mort, accordez-moi de me consacrer à ses funérailles, accordez-moi de consacrer mon temps à mes souffrances, de me familiariser avec mon malheur", etc. Ensuite tu diras: "allons, portons tous des libations sur le tombeau, l'un au nom de ses enfants, un autre au nom de son épouse, un autre au nom de son père. Lui seul, citoyens, a pris des risques pour nous tous, lui seul a écarté de nous le fléau. Cet homme est plus fortuné que son père, cet homme est vraiment heureux ». Ensuite : « ornons sa tombe de telle et telle façon, écrivons qu'il est notre bienfaiteur, notre sauveur, notre rempart contre le malheur. Inscrivons sur le tombeau ce que fut ce fléau, pour que le souvenir de son bienfait soit préservé pour les générations futures, pour que tous l'estiment, pour qu'ils se souviennent des honneurs que mon enfant a reçu après sa mort ». Ensuite tu diras : "Hâtons-nous de le consacrer propice et bienveillant par les honneurs et les cérémonies funèbres ». En effet tu amèneras grâce à de tels arguments que: "si vous me destituez, mon fils sera clairement peiné ». Tu diras donc: "Les défunts conservent une forme de perception après la mort, ils savent tout, observent ce qui se passe. Il se trouve assurément dans cette cour de justice, et voit les votes de chacun d'entre vous ». Ensuite adresse ton discours à l'enfant, conclus l'épilogue par un vœu : «allons, mon fils, toi qui fus le plus courageux face au danger, toi qui as surmonté toutes les épreuves grâce à ta grandeur d'âme, que tu te tiennes face à ce tribunal, ou que tu nous observes d'en haut : puissestu toujours garder le fléau éloigné du peuple, le malheur éloigné de la cité, et procure- 
nous ce qu'il y a de meilleur, pour que tu surpasses par tes faveurs pour le peuple tous les bienfaiteurs qui t'ont précédé ».

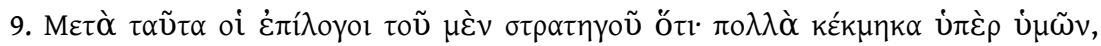

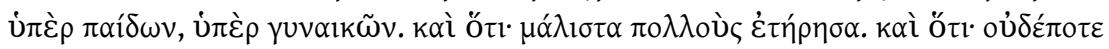

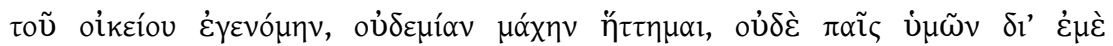

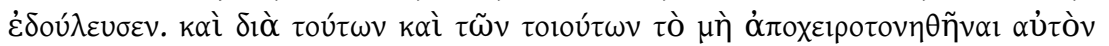

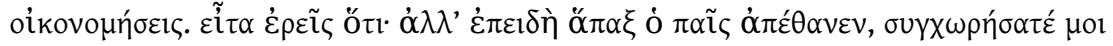

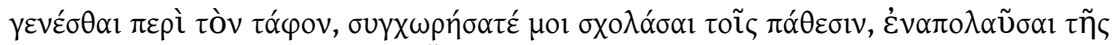

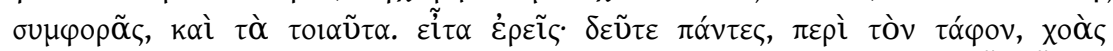

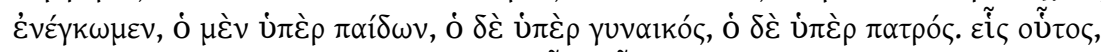

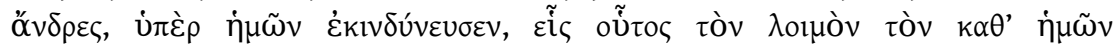

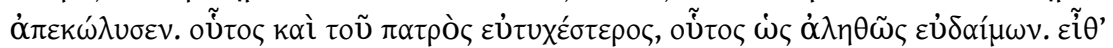

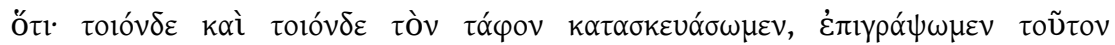

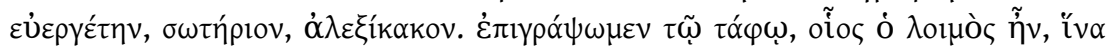

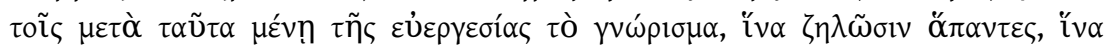

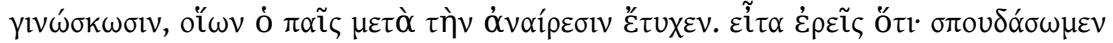

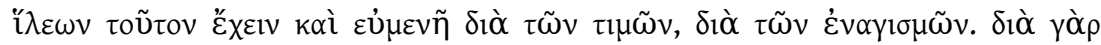

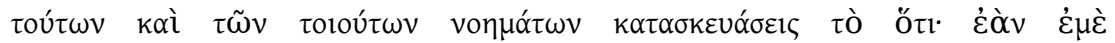

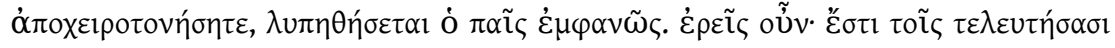

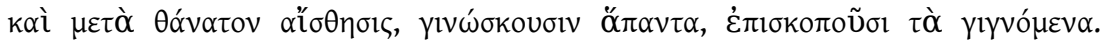

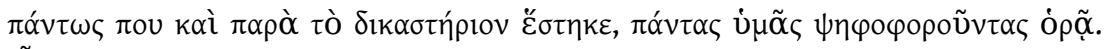

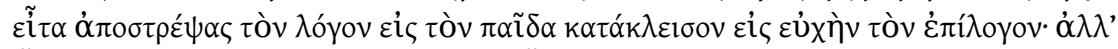

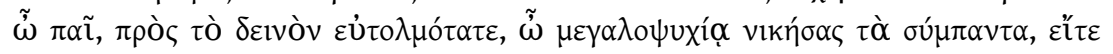

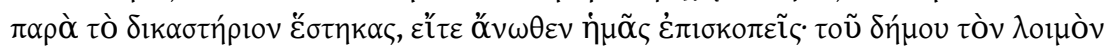

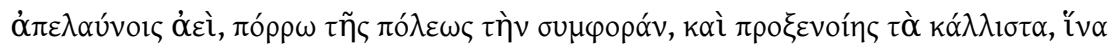

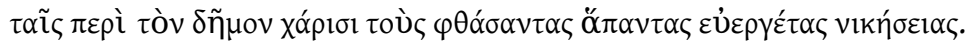

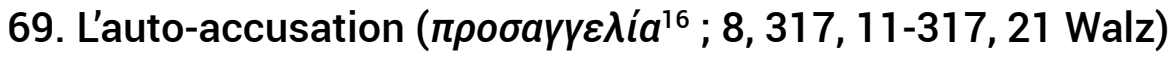

Le dieu a révélé à un homme aveugle qu'il retrouverait la vue si son enfant se sacrifiait pour lui. Après avoir appris l'oracle, l'enfant s'est suicidé. Le père a retrouvé la vue et après cela, il se rend à la justice.

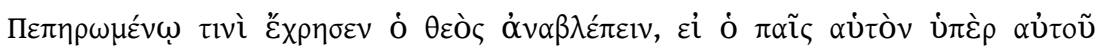

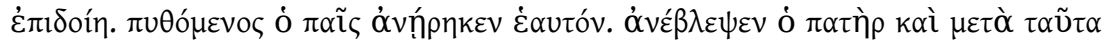
$\dot{\varepsilon} \alpha u t o ̀ v \pi \rho \circ \sigma \alpha \gamma \gamma \varepsilon ́ \lambda \lambda \varepsilon$.

L'exposé en faveur de l'enfant comprendra sa naissance avec emphase, l'émotion de l'enfant, sa mort. Tu établiras aussi la vertu de l'enfant et sa disposition vis-à-vis de son père. Première antithèse : tu n'as rien fait qui mérite la mort. Deuxième : l'enfant a obéi à l'oracle. Troisième : il a fait cela pour toi. Quatrième : l'enfant a obtenu la gloire. Cinquième : il fallait respecter l'oracle. Sixième : beaucoup d'autres ont perdu leurs enfants. Épilogue.

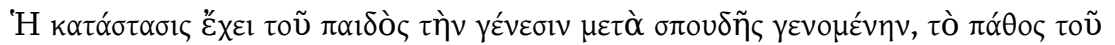

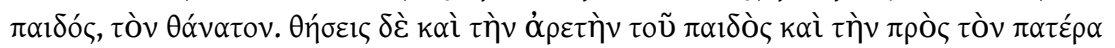

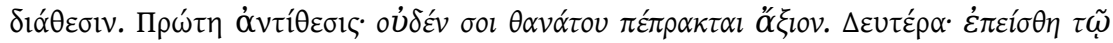

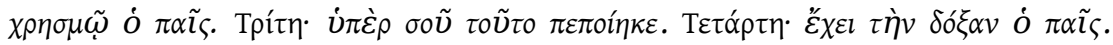

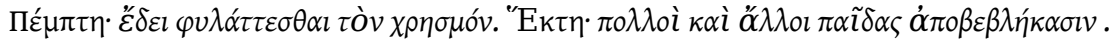

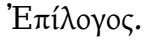




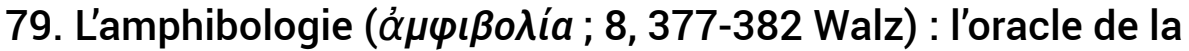 muraille de bois}

1. L'amphibologie se divise semblablement aux autres [états de la cause légaux] : par l'intention : qu'a-t-il plus voulu dire? «Je cède tous mes biens à Leon ou je cède mes biens à Pantaleon? " Ensuite quant à la matière des oppositions qui s'y appliquent. Et parfois aucune opposition n'apparaît, mais parfois aussi on divise en fonction des lieux : «ceci est plus utile pour vous; et ceci est plus réaliste que cela ». Ainsi dans l'exemple suivant: Zeus qui voit au loin donnera à Tritogénie une muraille de bois. L'un conseille les navires, l'autre conseille de fortifier l'acropole d'une muraille de bois ${ }^{17}$. L'amphibologie contient en elle-même la lettre établie par la conjecture. Ensuite, à ce stade, soit la question s'arrête ${ }^{18}$, car la matière n'a aucune opposition, soit, comme dans les autres cas, les oppositions se dégagent à partir des arguments des états de cause rationnels et de l'état pragmatique, et parfois aussi, comme ici, à partir des lieux: «ceci est plus profitable que cela; ceci est plus réaliste que cela »; etc.

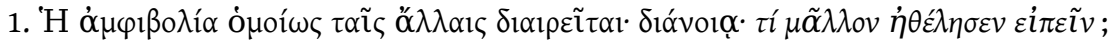

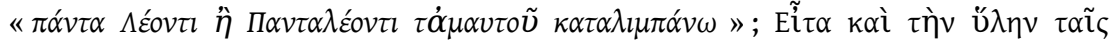

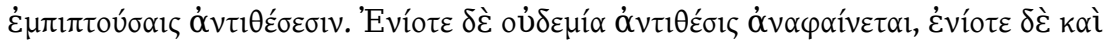

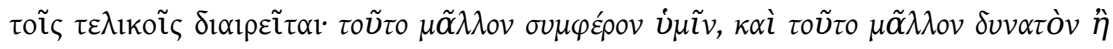

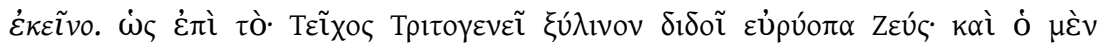

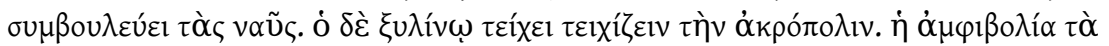

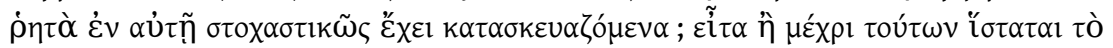

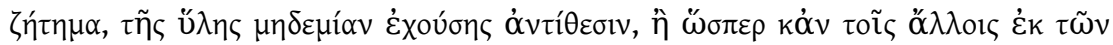

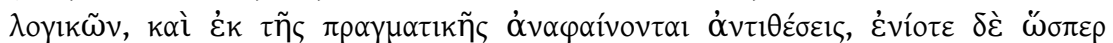

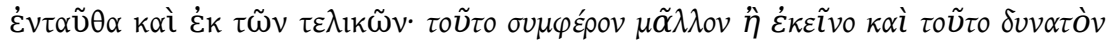

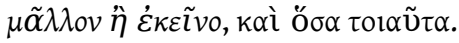

2. Par exemple, pour la question considérée, après l'exposé, dont la division est claire (les guerres Médiques, la peur ambiante, ensuite la question au dieu, ensuite l'oracle), tu diras d'emblée que le dieu parlait des trières: les trières constituent en effet la muraille de bois.

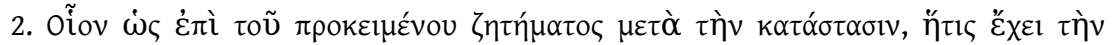

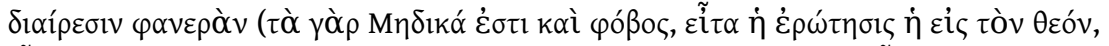

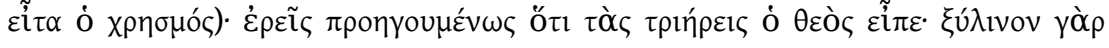

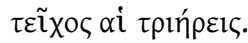

3. Ensuite, tu établiras la lettre à partir de l'opposition : « le Pythien, dit-il, n'a pas parlé des trières, mais il a dit de fortifier l'acropole grâce à une muraille de bois. Et en effet elle était auparavant fortifiée par une muraille de bois». La réfutation conjecturale : "le dieu n'a pas dit cela: en effet, cette muraille ne nous est utile en rien pour la guerre ». Et le développement de la réfutation est évident: «en quoi en effet cette muraille nous serait utile si la mer perdue, et les ports détruits, et si d'autres événements similaires se sont produits?» Ensuite, décrédibilisant la muraille de l'acropole, avance: «il n'est pas possible qu'elle résiste à une telle puissance du barbare. À quoi peut-elle résister, au feu ? aux machines de guerre? ? etc.

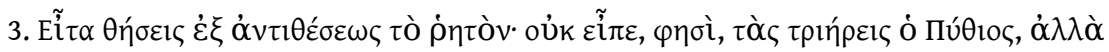

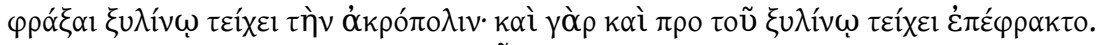

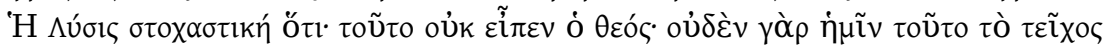

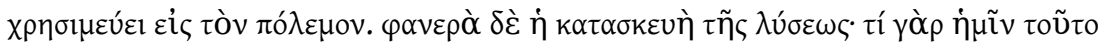

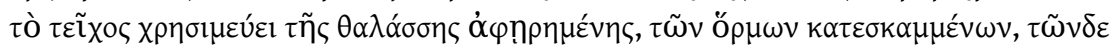

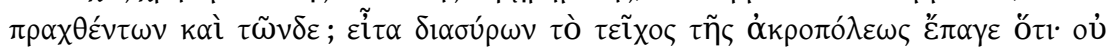




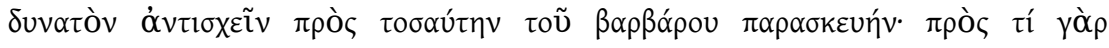

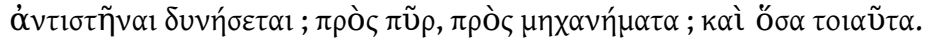

4. Après cela tu poseras encore une autre opposition : "mais équiper des trières est difficile, et cause de nombreux soucis", etc. Et la réfutation: "il ne faut pas se demander si c'est difficile mais si cela nous permettra de prendre le dessus sur le barbare ", etc.

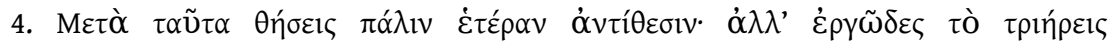

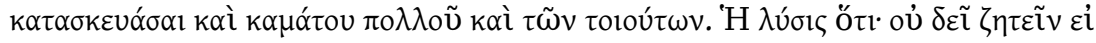

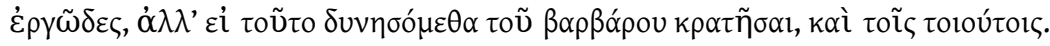

5. L'épilogue comprend l'exhortation pour les navires, de vives descriptions des combats et de nombreuses conjectures : les dieux seront nos alliés au combat; Athéna depuis notre cité, Déméter et Iacchos depuis Éleusis, et depuis Delphes, le Pythien, etc. $\mathrm{Tu}$ diras aussi quelle sera la situation après la victoire, et que: tous les Grecs reconnaîtront notre sagacité, et à quel point nous aurons de l'esprit, etc.

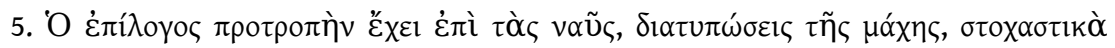

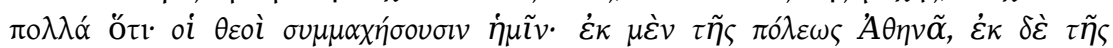

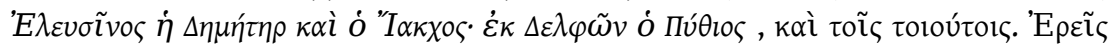

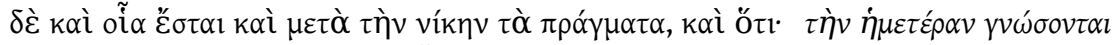

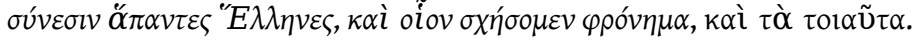

\section{NOTES}

1. Ces thèmes étaient suffisamment répandus pour être épinglés par Quintilien (Institution oratoire 2, 10, 4-5) ou Pétrone (Satyricon I, 2-3).

2. Pour une introduction à Sopatros, voir en particulier D. INNES et M. WINTERBOTTOM, Sopatros the

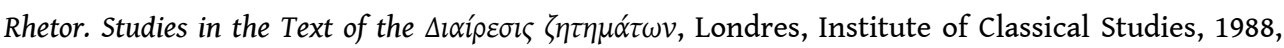
p. 1-20. Cette présentation de l'auteur et de son œuvre est due à M. Winterbottom et a été reprise dans l'ouvrage M. WINTERBOTTOM, Papers on Quintilian and Ancient Declamation, édité par A. Stramaglia, Fr. Romana Nocchi et R. Russo, Oxford, Oxford University Press, 2019, p. 135-160.

3. La $\sigma v \gamma \gamma v \omega ́ \mu \eta$, ou «demande de pardon » constitue, dans la théorie des états de la cause, une sous-catégorie d'opposition ( $\alpha \dot{v} \tau i ́ \theta \varepsilon \sigma l \varsigma)$. Pour Hermogène, ce cas se produit lorsque l'accusé reconnaît les faits, reconnaît être en faute, mais rejette la responsabilité de son acte sur un élément extérieur, qui ne peut toutefois être poursuivi en justice (si tel était le cas, il y aurait report d'accusation). Hermogène (Les états de la cause II, 8 = 39 Rabe) prend pour exemple un énoncé inspiré par le cas des généraux athéniens à la bataille des Arginuses : en raison d'une tempête, les généraux n'ont pas pu récupérer les corps des soldats morts durant le combat et ont été condamnés à mort. Ce thème semble avoir été très populaire auprès des rhéteurs de l'Antiquité (voir S.A.STEPHENS, "The 'Arginusae' Theme in Greek Rhetorical Theory and Practice", The Bulletin of the American Society of Papyrologists 20, 1983, p. 171-180). Sopatros privilégie un critère quelque peu différent : il y a transfert d'accusation si l'accusé impute la responsabilité de l'acte à un être ou un élément extérieur à sa personne, et demande de pardon s'il invoque un élément intérieur qu'il n'est pas possible de maîtriser (comme l'amour, la douleur ou la colère). Voir à ce propos M. Weißenberger, op. cit., p. 258-259. 
4. On trouve un thème similaire dans les petites déclamations du Ps.-Quintilien (326: «Legati filius uictima pestilentiae »). Il rappelle naturellement le sacrifice de Ménécée, fils de Créon, qui choisit de se sacrifier pour la ville de Thèbes alors que son père, alors intendant, refusait d'offrir la vie de son enfant pour le salut de la cité (C. АMIECH, « Le sacrifice de Ménécée : sa place, son rôle dans les Phéniciennes d'Euripide", L'information littéraire 60, 2008, p. 22-29; R. REBUFFAT, «Le sacrifice du fils de Créon dans les Phéniciennes d'Euripide », Revue des Études Anciennes 74, 1972, p. 14-31). Pensons également à la $3^{\text {ème }}$ suasoire de Sénèque le Rhéteur (« Deliberat Agamemnon an Iphigeniam immolet negante Calchante aliter navigari fas esse »).

5. Sopatros se concentre ici sur le développement de la défense, bien qu'il signale également certaines stratégies mobilisées par l'accusation et la manière d'y répondre.

6. Le tribunal s'assurait que le magistrat élu remplissait bien les conditions administratives inhérentes à sa fonction (âge, citoyenneté, charges précédentes) et examinait sa conduite et ses convictions politiques. Voir M. H. HANSEN, La Démocratie athénienne à l'époque de Démosthène, Paris, Éditions Tallandier, 2009, p. 255-257.

7. Les descriptions, ou ekphraseis, étant propices à « mettre sous les yeux » (c'est ainsi que ce type de description est définie, notamment, dans les manuels de progymnasmata; voir Ælius Théon, Prog. 7, 118 Patillon; Ps.-Hermogène, Prog. 10, 202 Patillon; Aphthonios, Prog. 12, 147 Patillon; Nicolaos, Prog 11, 67 Felten), l'orateur doit se garder de faire revivre ces souffrances à ses juges. Sur ce passage en particulier, voir R. WEBB, Ekphrasis, Imagination and Persuasion in Ancient Rhetorical Theory and Practice, Farnham, Ashgate, 2009 [2012], p. 143.

8. Cette première partie correspond à ce qu'Hermogène appelle la proposition du cas ( $\pi \rho \circ \beta 0 \lambda \eta ́)$. Il s'agit de rendre claire la position de l'accusation ou de la défense. Traditionnellement, elle se termine par amplification (si l'on suit ce que dit Hermogène, VI, 4). On voit ici quelle sera la ligne de défense du général : il a été contraint d'agir de la sorte par une force supérieure.

9. Il s'agit de contester le bien-fondé du chef d'accusation en s'appuyant sur sa définition. La pertinence de faire intervenir la définition dans les oppositions a fait l'objet de débats parmi les rhéteurs (voir en particulier le long développement proposé par Georges d'Alexandrie, Études sur les États de la cause d'Hermogène, XL, 15-40). Dans le cas d'une opposition, il s'agit avant tout d'une requalification $\mathrm{du}$ cas (Georges d'Alexandrie, XL, 13-14; Sopatros, Commentaire sur l'Art d'Hermogène, VI, 2,2) En l'occurrence, le général ne nie pas qu'avoir délibérément omis une partie de la réponse oraculaire pour sauver son fils constitue une faute envers la cité.

10. M. Patillon privilégie, dans ses traductions d'Hermogène et ses commentateurs, l'expression « examen des faits ». Proche de la narration, le compte-rendu des événements s'en distingue par le fait qu'il s'agit d'une démonstration argumentée qui s'appuie sur les lieux et les circonstances, en particulier, dans un cas comme celui-ci, sur l'intention. Georges d'Alexandrie précise encore : «bien que l'un et l'autre contiennent une narration et une amplification, dans la proposition la narration occupe une place plus importante que l'amplification, et dans l'examen des faits au contraire l'amplification a plus d'importance que la narration » (XL, 46 ; trad. : M. PATILLON, Paris, Les Belles Lettres, CUF, 2018). Ce point est davantage lié à l'accusation.

11. Pour contrer le compte-rendu des événements, l'accusé fera valoir son intention : il n'a pas agi contre la cité, mais pour protéger son fils, mû par une force qu'il ne pouvait contrôler (la nature). Sur la succession de ces deux points, voir Hermogène, Les états de la cause, VI, 4-5.

12. Ou " définition violente " (Patillon). Dans son édition des États de la cause d'Hermogène dans la Collection des Universités de France, M. Patillon donne de ce qualificatif la justification suivante : «Le qualificatif 'violente' vient de ce que la définition employée ici force les choses et va à l'encontre de la réalité obvie » (op. cit., p. 176, n. 5). Hermogène choisit d'expliquer en quoi cela consiste par un exemple (Les états de la cause, VI, 10, trad. M. PATILLON, CUF, 2009 ; modifiée) : « lors d'un siège et d'une disette le général a été d'avis de faire une sortie et de livrer bataille, mais cet avis n'a pas triomphé ; en secret il a ouvert une brèche dans le rempart et la sortie s'est faite, suivie de la victoire; plus tard il répond à une accusation à titre public. La définition 
forcée : ‘plutôt qu'une victoire, n'est-ce pas la prise et la destruction de la ville ?' Et l'accusé en sens inverse : 'plutôt qu'ouvrir une brèche dans le rempart, n'est-ce pas le relever alors qu'il était tombé ?' »

13. C'est-à-dire les éléments sur lesquels l'orateur va pouvoir s'appuyer pour sa démonstration.

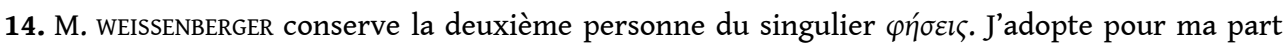
une correction suggérée par M. Winterbottom (dans D. INNES et M. WINTERBOTTOM, op. cit., p. 179). En effet, Sopatros anticipe ici un argument de l'accusation dans le but de conseiller la défense sur la meilleure manière de le contrer. Or, les deuxièmes personnes de ce texte renvoient à la personne en charge de la défense, tandis qu'il est fait référence à l'accusation à la troisième personne du singulier. Il me semble donc opportun de marquer, ici aussi, ce changement de

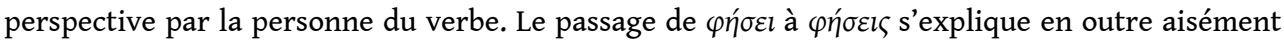
par la présence de la préposition $\varepsilon i \zeta$.

15. Ce point correspond, dans la structure du discours, à l'antilepse, c'est-à-dire la réponse de l'accusé à la métalepse de l'accusateur. Toutefois, dans le cas d'une demande de pardon, dans la mesure où l'accusé ne conteste pas avoir commis une faute, celle-ci se voit réduite.

16. Bien que l'expression signifie littéralement "l'auto-accusation", ces cas concernent des demandes d'autorisation de suicide. Sur le sens de cette expression, voir notamment R.J. LOENERTZ, « ПРОГАГГЕ $\Lambda$ ЕIN 'EAYTON », Byzantion 29/30, 1959-1960, p. 1-6 ; ou D. Russell, Greek Declamation, Cambridge, Cambridge University Press, 1983, p. 35-37, pour un commentaire sur sa signification dans le contexte spécifique de la déclamation.

17. Voir Hérodote VII, 140-145 pour un compte-rendu de la délibération qui a suivi ce célèbre oracle.

18. Cela signifie que la question est mal formée car elle n'est pas étayée par des circonstances (cf.

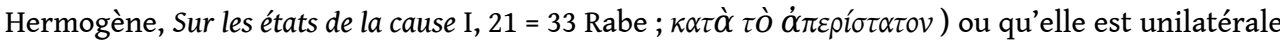

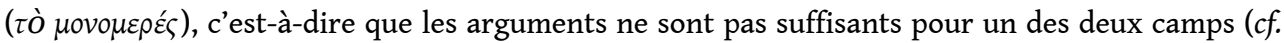
Hermogène, Sur les états de la cause I, 14 = 32 Rabe).

\section{AUTEUR}

\section{JULIE DAINVILLE}

Université libre de Bruxelles/ Université de Lausanne 